\title{
Family medicine in South Africa: exploring future scenarios
}

\author{
R Masha* and K Von Pressentin $^{\mathrm{a}}$ (D) \\ ${ }^{a}$ Division of Family Medicine and Primary Care, Stellenbosch University, Cape Town, South Africa \\ *Corresponding author, email:rm@sun.ac.za
}

This paper reports on a workshop held at the 19th National Family Practitioners Conference in August 2016. The aim of the workshop was to describe possible future scenarios for the discipline of family medicine in South Africa and identify possible options for action. The workshop led a group of 40 family physicians from academic, public and private sector settings through a scenario planning process developed by Clem Sunter and Chantell Ilbury. After an overview of the current situation the participants reached a consensus on the rules of the game, key uncertainties, future scenarios and options for action. The main message was that the South African Academy of Family Physicians as a professional body needs to take a stronger role in advocating for the contribution of family medicine to government, health managers and the public.

Keywords: family medicine, family physicians, primary care, scenario planning, South Africa

\section{Introduction}

This article reports on the findings of a workshop at the 19th National Family Practitioners Conference in August 2016 that used the scenario planning approach of Clem Sunter and Chantell Ilbury to explore future possible scenarios for family medicine in South Africa. ${ }^{1}$

Before considering the possible future scenarios it is important to briefly evaluate our current situation. As a discipline family medicine is engaged with both the health system and the education system. ${ }^{2}$ These two systems are interdependent as the educational system provides the workforce for the health system, while the health system provides the context of training and eventual place of employment. Both should be aligned with the health needs of the population to ensure that the relevant services are provided and that health care workers are appropriately trained.

\section{Current education system and family medicine}

In terms of the education system, family medicine is now well embedded in both undergraduate and postgraduate medical curricula at all medical schools in South Africa and provides community-based decentralised training. At an undergraduate level family medicine is also responsible for the training of clinical associates: mid-level doctors. At a postgraduate level we have agreed nationally on learning outcomes for a two-year Diploma aimed at primary care doctors ${ }^{3}$ and a four-year Master of Medicine degree $^{4}$ aimed at training family physicians. The training of clinical nurse practitioners, however, who provide most of the primary care in the public sector, is much less engaged with family medicine.

Registrars are trained to be family physicians over a period of four years and we have succeeded in accrediting training programmes at all nine medical schools with a national exit examination under the auspices of the South African College of Family Physicians. Training is accredited by the Health Professions Council of South Africa and co-ordinated by the South African Academy of Family Physicians. All registrars must also complete a research assignment and a minimum of three years' training in a registrar post. Training is outcome and competency based and aims to deliver a family physician who is a competent clinician in the district hospital and primary care, able to act as a consultant to and build the capacity of the rest of the health care team, able to lead clinical governance in order to improve the quality of care, and able to support community-orientated primary care, such as the ward-based outreach teams. ${ }^{5}$ In many cases the family physician must also be a competent clinical trainer and teacher to medical students, interns, clinical associate trainees and registrars. A wide range of relevant clinical skills have been identified and agreed upon nationally. ${ }^{6}$

Registrar training started in 2008 and the number of new registrars entering the programmes reached a peak of around 70 by 2012 (personal communication, National Education and Training Committee of the SA Academy of Family Physicians). Since then the number of new registrars across the country has fallen to around 40 per year over the nine programmes. This is in part due to moratoriums on all registrar posts in two provinces, but may also be due to a lack of suitable applicants. Outputs from the programmes has also been relatively small, with around 18-20 new family physicians per year nationally. Outputs have varied significantly between programmes with Stellenbosch, Sefako Magatho, KwaZulu-Natal and Free State universities contributing the majority of new family physicians.

Overall the strengths of the educational system have been cooperation between the programmes and consensus on the roles, competencies, learning outcomes and clinical skills required. Clinical training has also been well supported by a nationally developed portfolio of learning as well as local textbooks. ${ }^{7,8}$ The launch of a new postgraduate Diploma in 2016 may also help prepare the pool of primary care doctors for the future re-engineered primary care system in South Africa. The integration of clinical associate training with the training of doctors is also a potential strength in terms of shared values and inter-professional collaboration.

On the other hand the weaknesses of the educational system include a mismatch between the numbers of registrars in the training programmes and the relatively small outputs, indicating significant bottlenecks, particularly with regard to the research 
assignment. The low numbers entering the programmes is also a weakness and there is also concern about the quality and commitment of those applying. Given that postgraduate training in the district health system is a new phenomenon there is a need to enhance the capability of family physicians as clinical trainers and to ensure they have the capacity in terms of time and resources to train effectively. In many areas there are few family physicians to act as trainers and to be role models that attract registrars. In some provinces there are very few registrar posts on offer.

\section{Current health system and family medicine}

Current health policy recognises a place for family physicians in the health system. The National Development Plan particularly highlights their role in clinical governance and leading quality improvement in the district health system. ${ }^{9}$ The National Human Resource Plan recognises the need to restore the place of the generalist doctor in primary health care teams ${ }^{10}$ and the Ideal Clinic policy recognises that every clinic should at least have access to a primary care doctor. ${ }^{11}$ The Human Resource policy also saw a role for the family physician in District Clinical Specialist Teams that focus on maternal and child health.

The Human Resource policy identified a human resource gap of 888 family physicians that were needed for the country, although this was based on data suggesting that the country already employed 521 family physicians in the public sector and 269 in the private sector in 2008. ${ }^{10} \mathrm{~A}$ research study in 2016 estimated that there were just over 200 family physicians employed in the public sector, suggesting that the data used by the Human Resource policy was unreliable (personal communication, unpublished data, Dr von Pressentin). The data from this study estimated that we have 0.035 family physicians per 10,000 population in the public sector and World Bank figures suggest an overall rate of 0.1 per 10,000 in all sectors. These rates can be compared with countries such as Brazil $(0.2$ per 10,000$)$, China (1.2 per 10,000$)$ or the UK and north America (4.0-12.0 per 10,000). ${ }^{12}$

The recently published white paper on National Health Insurance also recognises a role for the family physician in district hospitals, although mistakenly seems to allocate this role to a Department of Family Medicine within such a hospital. ${ }^{13}$ It is clear that while policy recognises the contribution of family physicians there is some confusion, misinformation and diversity within and between different policies.

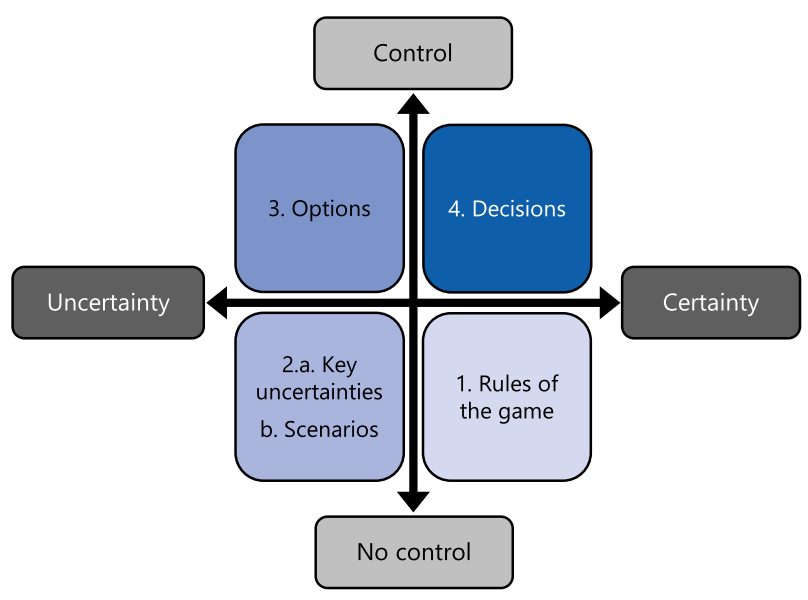

Figure 1: Conceptual model for scenario planning. Source: Sunter and Ilbury. ${ }^{1}$
Within the private sector family physicians have not been fully recognised as although they may receive a practice number as a 'specialist' in lieu of their postgraduate training the medical schemes are not remunerating them as such or recognising their skills set.

The strengths of the health system with regard to family medicine are a clear commitment to universal health coverage, improving primary health care, enhancing community-orientated primary care and building leadership capacity. There is also a clear recognition of the need for family physicians as part of this and some provinces, most notably the Western Cape, have started to go to scale with employing family physicians through the district health system. Weaknesses with regard to family medicine include confusion regarding the role of family physicians at district hospitals, uncertainty about their place in the primary care team and variability in deployment between provinces.

\section{Scenario planning process}

The scenario planning process involved a group of 40 people attending the national conference who were divided into groups of 6-7 people. The attendees included family physicians working in the public and private sectors as well as teachers and academic staff from the universities. After a brief overview and discussion of the current situation the groups worked through a series of steps according to the conceptual framework illustrated in Figure 1.

The model is structured around a vertical axis representing the degree of control an organisation has over the issues and a horizontal axis representing the degree of certainty that the organisation has regarding the issues. These axes therefore define four quadrants and scenario planning follows a step-wise process that starts in the bottom right-hand quadrant.

\section{Rules of the game}

The bottom right-hand quadrant represents the 'rules of the game' over which we have no control, but which are clearly identifiable and define the 'playing field' in which we can operate or manoeuvre. Rules may be explicitly stated in law or policy for example or implicit in the organisation or broader society. Sometimes they may even be contradictory. Each group was asked to brainstorm and identify 4-6 important rules that govern the future of family medicine in South Africa.

\section{Key uncertainties}

Following this the groups were asked to identify 2-3 'key uncertainties' in the bottom left-hand quadrant that represented factors that are uncertain and over which we have little control. Key uncertainties were defined as forces that could be clearly identified and would potentially impact on the discipline of family medicine and could be seen as either opportunities or threats.

\section{Scenarios}

Given the 'rules of the game' and the 'key uncertainties' groups were then asked to imagine 2-3 possible future scenarios. These scenarios were not seen as predictions, but as possible future directions or pathways. Groups were asked to give each scenario a title and to describe the essential characteristics in 2-3 sentences.

\section{Options}

Participants were finally asked to try to identify the options for action as per the top left-hand quadrant. Options for action were seen as opportunities within our control that are achievable although it is not yet certain which options will be implemented. The groups imagined that they were defining options for the South African Academy of Family Physicians. The 
workshop did not address the final quadrant, which requires the organisation to decide which of the options will actually be implemented.

After each step described above the groups shared their ideas with the other participants and the ideas were collated and synthesised by the facilitators. The findings presented here represent the consensus of all groups that participated in the workshop.

\section{Rules of the game}

Groups identified the following rules that define the playing field for family medicine:

- Globally we are committed to the Sustainable Development Goals, which include a commitment to universal health coverage and primary health care. ${ }^{14}$

- Nationally we have defined a development plan that includes development of the health system and a contribution from family physicians. ${ }^{9}$

- We face a quadruple burden of disease within which chronic diseases are increasingly important. ${ }^{15}$

- Family medicine embraces a set of generalist principles that are not the same as other specialist disciplines. ${ }^{16}$

- The health system is divided into inequitable private and public health sectors and the roles of the family physician differ.

- The Department of Health in the public sector decides on the available posts for registrars in training and family physicians and operates within a resource-constrained environment.

- This constrained environment of the public sector poses ongoing challenges between balancing the training needs of family medicine registrars and meeting the service delivery requirements.

- The medical schemes in the private sector determine whether family physicians are recognised within the staff mix.

- Family physicians must be trained to meet the nationally defined learning outcomes and to be competent across a multifaceted set of roles and competencies that go beyond the purely clinical.

- The Health Professions Council of South Africa decides on the registration requirements for family physicians.

- South Africa has recognised the new cadre of clinical associates to work in the health system.

\section{Key uncertainties}

Key uncertainties included:

- the economic position of the country and the ability to expand the health care workforce to include more family physicians;

- the implementation of National Health Insurance at a national level;

- clarification of the roles and contribution of family physicians to the health system at a national level and expression of this consistently in policy;

- the variable implementation of policy by provinces and whether this would include a commitment to deploy family physicians and create career pathways;

- public awareness of and support for family physicians versus medical officers and general practitioners without postgraduate training;
- ability of the training programmes to deliver a supply of family physicians at sufficient scale for the needs of the country;

- commitment of the district health system to the training of family physicians in terms of registrar posts and capacity for training;

- realisation of the family physicians' contribution in all the envisaged roles that they are trained for;

- clarification of the role of the family physician in facility-based and community-based multidisciplinary primary care teams;

- commitment to recruit and retain family physicians in rural and remote areas;

- relationship between Independent Practitioner Associations and other entities in the private sector.

\section{Scenarios}

Three scenarios were outlined:

\section{Pot of gold}

The health of South African society is improved. In this scenario National Health Insurance is implemented and enables Universal Health Coverage with integration of resources in the public and private health care sectors. The principles of family medicine, primary health care and generalism are at the heart of the health system. Policy-makers are clear about the roles and contribution of family physicians. Primary health care is strengthened and family physicians work in multidisciplinary teams to support facility-based and community-based services. Family physicians are also employed at district hospitals, especially in more rural areas. There is a move towards ensuring that all doctors working as generalists have postgraduate training. As a result the health and educational systems collaborate to ensure that quality training programmes are embedded in the district health system with career pathways for family physicians.

\section{Business as usual}

The health system continues to struggle with a lack of resources and poor quality in the public sector. Patients vote with their feet whenever possible to seek help elsewhere. Family medicine training programmes continue to deliver small numbers of family physicians and struggle to provide quality training through capacitated clinical trainers in the district health systems. Key uncertainties remain unresolved and while the situation goes up and down the issues essentially remain the same. Family physicians are not integrated into the health system, particularly in rural and remote areas.

\section{Times are dark}

The economic constraints worsen and National Health Insurance is poorly implemented and 'bleeds us dry'. Family physicians leave for greener pastures in the private sector or overseas and there are fewer clinical trainers available. Registrars are few and worry about having a career pathway after qualifying and their ability to support their families. The Department of Health decides that they will not support the deployment of family physicians and focuses on other areas.

\section{Options}

The workshop considered the options available to the profession and to the Academy given the above scenarios. The following options were identified: 
- The Academy as our professional body could engage more proactively with policy-makers in the Department of Health and the Treasury, and to develop skills in advocacy to communicate our vision more clearly. In particular to engage with the process of re-engineering primary health care and national health insurance. The voice of rural doctors and contribution of family medicine in rural areas needs to be more clearly articulated.

- The Academy could ensure that research evidence of the contribution of family medicine to the health system is showcased and communicated.

- The Academy could more confidently make the case for family medicine to the public through a marketing campaign and the general media.

- The Academy could use their journal, the South African Family Practice Journal, to promote the viewpoint of the discipline.

- The Academy could actively foster the next generation of leaders and ensure that new ideas and people are involved in leading the profession.

\section{Conclusion}

This article has outlined the process of a scenario planning workshop held at the 19th National Family Practitioner Conference and represents a consensus of the participants. The workshop outlined three possible future scenarios and presented the Academy leadership with a number of options, which broadly speaking ask the Academy to take a more active role in advocating and communicating for the discipline in South Africa. The final quadrant of the scenario planning model rests with the Academy itself and requires that key decisions be made and implemented based on the suggested options.

Acknowledgements - The authors are grateful to all the people who participated in the workshop at the 19th National Family Practitioners Conference in August 2016.

Conflict of interest - The authors report no conflict of interest and received no financial support for the work reported on in this article.

\section{ORCID}

K Von Pressentin (D) http://orcid.org/0000-0001-5965-9721

\section{References}

1. Sunter C, Ilbury $C$. The mind of a fox: scenario planning in action. Cape Town: Human \& Rousseau Tafelberg; 2011.

2. Frenk J, Chen L, Bhutta $Z$, et al. Health professionals for a new century: transforming education to strengthen health systems in an interdependent world. Lancet. 2010;376:1923-58. http://dx.doi. org/10.1016/S0140-6736(10)61854-5

3. Mash R, Malan Z, von Pressentin K, et al. Strengthening primary health care through primary care doctors: the design of a new national Postgraduate Diploma in Family Medicine. S Afr Fam Pract. 2015. doi: 10.1080/20786190.2015.1083719.

4. Couper I, Mash B, Smith S, et al. Outcomes for family medicine postgraduate training in South Africa. S Afr Fam Pract. 2012;54(6):5016. http://dx.doi.org/10.1080/20786204.2012.10874283

5. Mash R, Ogunbanjo G, Naidoo SS, et al. The contribution of family physicians to district health services: a national position paper for South Africa. S Afr Fam Pract. 2015;57(3):54-61.

6. Couper I, Mash B. Obtaining consensus on core clinical skills for training in family medicine. S Afr Fam Pract. 2008;50(6):69-73.

7. Mash B, editor. Handbook of family medicine (3rd ed.). Cape Town: Oxford University Press; 2011.

8. Mash B, Blitz J, editors. South African family practice manual (3rd ed.). Cape Town: Van Schaik; 2014.

9. Government of South Africa. Chapter 10: promoting health in the National Development Plan - 2030. Pretoria: Government of South Africa; 2013. p. 330-51.

10. National Department of Health. Human Resources for Health, South Africa, 2030. Pretoria: NDoH; 2011.

11. Department of Health. Ideal clinic components and definitions. Pretoria: National Department of Health; 2015.

12. The World Bank. Data [cited June 19, 2015]. Available from: http:// data.worldbank.org.

13. National Department of Health. National Health Insurance in South Africa: policy paper. Pretoria: NDoH; 2016.

14. United Nations. Sustainable Development Goals [cited October 8, 2016]. Available from: http://www.un.org/sustainabledevelopment/ sustainable-development-goals/

15. Bradshaw D, Norman R, Schneider MA. Clarion call for action based on refined DALY estimates for South Africa. S Afr Med J. 2007;97(6): 438-40.

16. Howe A. Medical generalism: why expertise in whole person medicine matters [cited 5 November, 2013]. London: RCGP; 2012. Available from: http://www.rcgp.org.uk/policy/rcgp-policy-areas/ / media/Files/Policy/A-Z-policy/Medical-Generalism-Why_expertise_ in_whole_person_medicine_matters.ashx. 